\title{
An Uncommon Case of Recurrent Midventricular Takotsubo Cardiomiopathy
}

\author{
Giovanna Chiqueto Duarte ${ }^{1 *}$, André Luiz Gargioni de Andrade ${ }^{1}$, Julio De Paiva Maia ${ }^{2}$ and Marcos Franchetti ${ }^{2}$ \\ ${ }^{1}$ Undergraduate at State University of Maringá (UEM), Brazil \\ ${ }^{2}$ Diagnostic Center of Parana (CEDIPAR), Maringá, Brazil
}

*Corresponding author: Giovanna Chiqueto Duarte, Undergraduate at State University of Maringá (UEM), Brazil.

Received Date: October 09, 2019

Published Date: October 16, 2019

\begin{abstract}
Recurrent Takotsubo Cardiomiopathy is a rare condition. Recent studies report an estimated recurrence rate of $4 \% 1$. We report a case of a postmenopausal woman with two episodes of midventricular variant of Takotsubo Cardiomiopathy in a period of one year.
\end{abstract}

Keywords: Recurrent takotsubo cardiomiopathy; Midventricular takotsubo; Myocardial infarction

Abbreviations: TC: Takotsubo Cardiomiopathy; LV: Left ventricle; MRI: Magnetic Resonance Imaging

\section{Introduction}

Takotsubo cardiomyopathy (TC) is a transient systolic dysfunction of the left ventricle (LV) occurring mainly in postmenopausal women after a stressful event [1]. It is associated with characteristic LV contraction patterns, typically apical dyskinesia and basal hyperkinesia [2]. However, several variants of TC have been described. The midventricular type is characterized by akinesis with or without ballooning of the midventricular segment and a hyperdynamic pattern of base and apex [3]. The exact pathophysiology of TC is still unclear, although several hypotheses, such as multivessel coronary spasm, microvascular impairment and direct catecholamine-mediated myocardial stunning, have been proposed [4]. Recurrent TC is uncommon. The reported recurrence rate of TC has ranged from 1 to $6 \% 1$. In this case report, we describe a 59-year-old female presenting a midventricular variant of TC and one-year recurrence after the first episode.

\section{Case Presentation}

A 59-year-old dyslipidemic woman was admitted in our hospital presenting typical chest pain, which started after a serious medical problem regarding her mother. Although the admission electrocardiogram showed no significant abnormalities, the initial set of cardiac biomarkers revealed elevated creatine kinase-MB
(14.9 ng/ml, normal < $4.3 \mathrm{ng} / \mathrm{ml}$ ), troponin I (3.4 ng/ml, normal $<0.4 \mathrm{ng} / \mathrm{ml}$ ) and myoglobin (116 ng/ml, normal < $107 \mathrm{ng} / \mathrm{ml}$ ) levels, raising the hypothesis of a non-ST-segment-elevation acute myocardial infarction. Urgent coronary angiogram was performed. Ventriculography revealed increased LV end-diastolic volume with associated ventricular mid portion akinesis of anterior and inferior walls (Figure 1), and no obstructive coronary disease or angiographic evidence of acute plaque rupture were found (Figure 2).

So, a midventricular TC was diagnosed. She had clinical improvement and was discharged four days later, assimptomatic, with medical treatment for heart failure. Three weeks after hospital discharge the patient underwent cardiac magnetic resonance imaging (MRI), which revealed resolution of the systolic disfunction and normalization of wall-motion abnormalities (Figure 3). One year later, after knowing about her mother's death, the patient returned to our hospital presenting the same previous symptoms and elevated levels of cardiac biomarkers. A new coronary angiography revealed non-significant atherosclerotic lesions and ventriculography pattern was similar to the previous one. Therefore, the patient was diagnosed with recurrent midventricular TC. Five months later, an echocardiogram showed total dyskinesia recovery. 

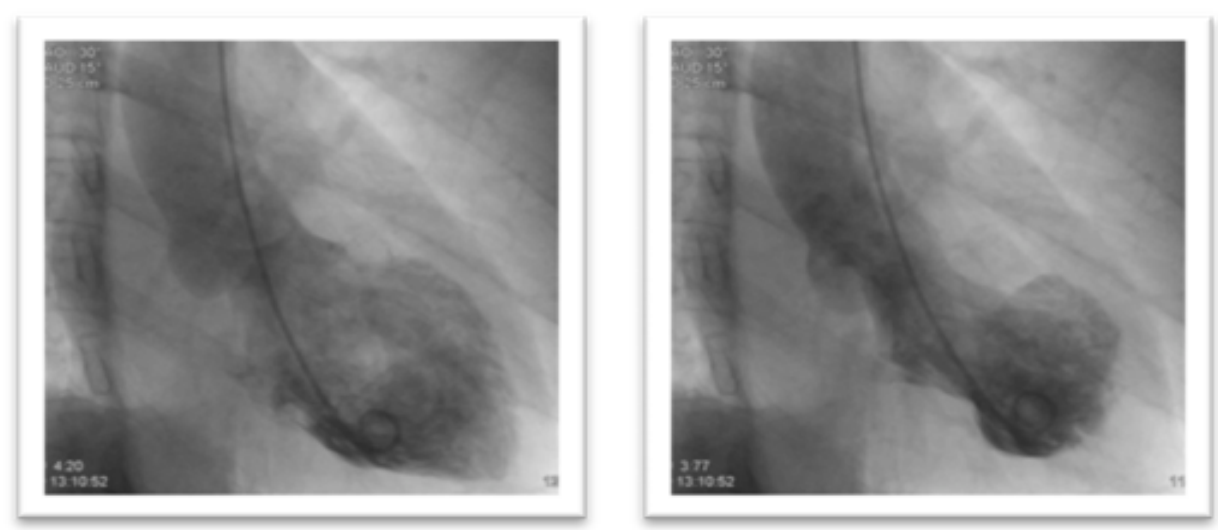

Figure 1: Ventriculography during the systole and diastole.
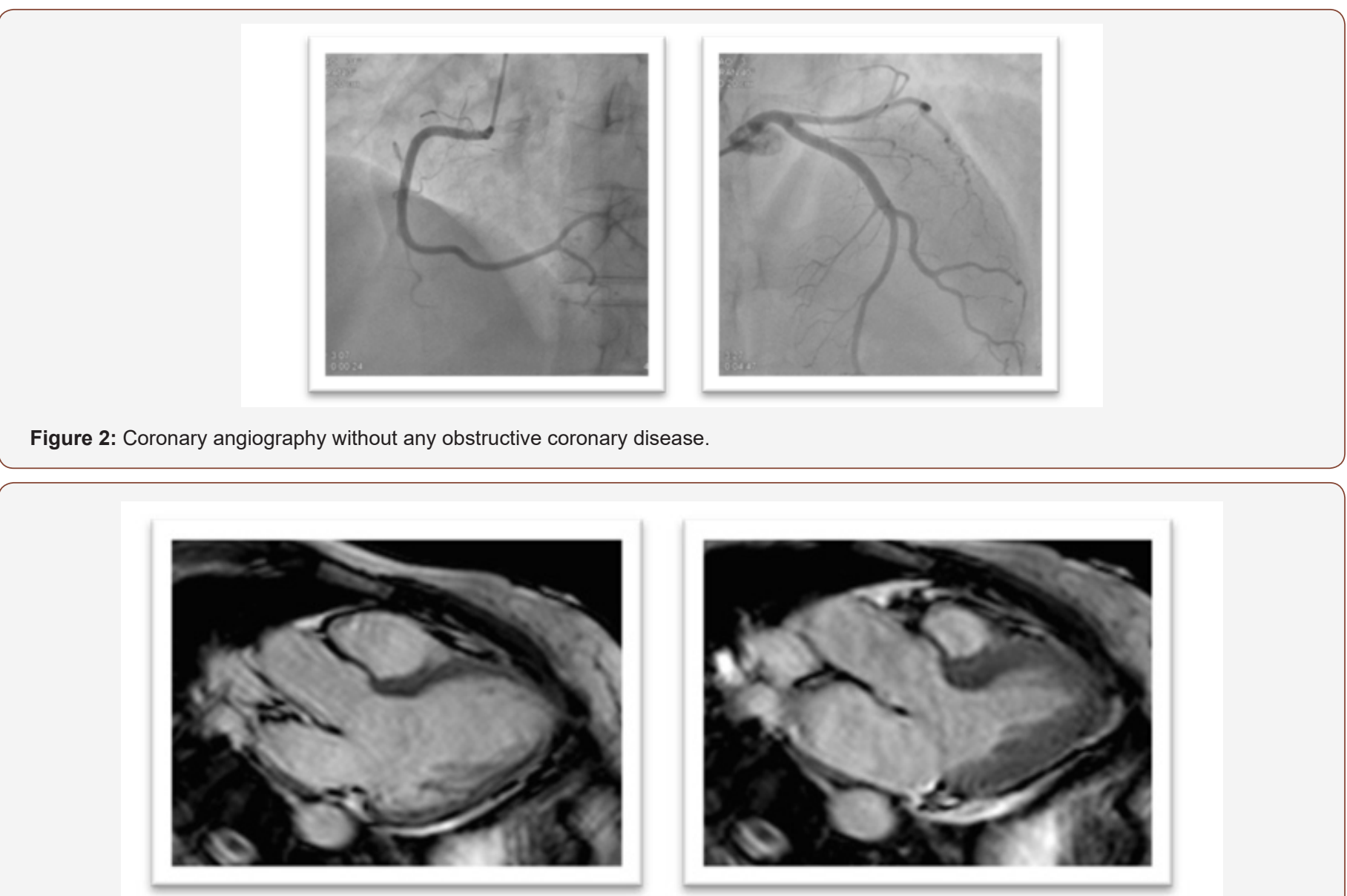

Figure 3: Cardiac MRI showing resolution of abnormalities.

\section{Discussion}

Takotsubo cardiomiopathy was originally described by Japanese authors in 1990s, as a cause of myocardial infarction with nonobstructive coronary arteries disease and characterized by severe LV disfunction that typically recovers spontaneously within days or weeks. The name was attributed to the appearance of the LV during the systole in ventriculography, which resembles a takotsubo, a pot used by Japanese fishermen to trap octopuses [5]. The exact pathogenesis of TC is still unknown. Several hypotheses, such as multivessel coronary spasm and microvascular impairment have been associated. However, emotional distress causing elevated levels of catecholamines has been proposed as the most underlying cause of this rare condition. Nevertheless, about one in five patients does not describe any form of stress preceding the onset of the condition [4]. In this case the patient related a medical problem regarding her mother before the first episode, and her mother's death preceding the second episode of chest pain. Most of the TC cases are observed in women. Studies have shown that almost $90 \%$ of patients with TC are postmenopausal women, with a similar prevalence across ethnic groups [4]. Patients typically present 
classical symptoms of myocardial infarction such as acute chest pain and dyspnea. Those findings are aligned to the present case report.

Takotsubo cardiomiopathy diagnosis is traditionally performed using the modified Mayo Clinic criteria, which require all the following to be met: (a) transient wall motion abnormalities of $\mathrm{LV}$ which extend beyond a single epicardial vascular distribution; a stressful trigger is often seen but not always present; (b) absence of obstructive coronary disease or angiographic evidence of acute plaque rupture; (c) new electrocardiographic abnormalities (either ST-segment elevation and/or T wave inversion) or modest elevation in cardiac troponin; and (d) absence of pheochromocytoma or myocarditis [6]. Our patient satisfied these criteria. Typically, patients with TC manifest modest increases of creatine kinase-MB and cardiac troponin concentrations compared to patients with ST-segment-elevation acute myocardial infarction. Of interest, in TC, there is a disparity between the degree of biomarker elevation and the extent of myocardial dysfunction observed at left ventriculography. In a minority of patients with TC, however, the elevation of biomarkers can be substantial, probably reflecting more severe myocardial damage [4]. In the present case there were increases in cardiac biomarkers in both episodes, which was consistent with ventriculography findings.

Coronary angiography did not show significant disease in both events. However, ventriculography revealed increased LV enddiastolic volume with associated ventricular mid portion akinesis of anterior and inferior walls. Therefore, the diagnosis of a TC could be confirmed. Cardiac MRI was performed weeks after the first case and an echocardiogram after the second one, revealing total recovery of the ventricular dyskinesia.
Recurrent TC is uncommon. Literature reports a recurrence rate of $1 \%$ to $6 \%$. Nonetheless, these data are inconsistent and limited. Recent European multicenter TC registry reported a recurrence rate of $4 \%$, with a predominance of a single recurrent event1. Our patient had recurrent TC presenting identical clinical pattern and similar echocardiographic findings compared to the first presentation.

\section{Acknowledgments}

The authors appreciate the contributions made by Afonso Shiozaki.

\section{Conflict of Interest}

No conflict of interest.

\section{References}

1. El-Battrawy I, Santoro F, Stiermaier T, Möller C, Guastafierro F, et al (2019) Incidence and Clinical Impact of Recurrent Takotsubo Syndrome: Results From the GEIST Registry. Journal of the American Heart Association 8(9): e010753.

2. Emily M Miner, Harini Gurram, Tennie Renkens, Julie L Welch (2017) Recurrent Takotsubo Cardiomyopathy: A Rare Diagnosis with a Common Emergency Department Presentation. Arch Med Pp. 9(4).

3. Yamamoto Y, Watari Y, Kobayashi K, Tanaka K (2010) Recurrent takotsubo cardiomyopathy can appear as transient midventricular ballooning syndrome. Journal of cardiology cases 1(3): e141-e143.

4. Pelliccia F, Kaski JC, Crea F, Camici PG (2017) Pathophysiology of Takotsubo syndrome. Circulation, 135(24): 2426-2441.

5. Khouri S, Imran N (2009) Stress cardiomyopathy (takotsubo cardiomyopathy). Clinical medicine Cardiology 3: 93-99.

6. Sharath Babu NM, Chacko ST, Chacko BR, Irodi A (2019) Recurrent Takotsubo cardiomyopathy in a postmenopausal Indian lady: Is there a pattern. Journal of postgraduate medicine 65(2): 112-115. 